\title{
Colaboração Institucional Entre Ministério Público, Poder Judiciário e os Profissionais de Psicologia das Políticas Públicas de Assistência Social e de Saúde - Limites Éticos e Possibilidades ${ }^{1}$
}

Diego Mendonça Viana ${ }^{2}$

\section{RESUMO}

trabalho objetiva discutir os diversos e complexos fatores que envolvem a colaboração interinstitucional entre Ministério Público (MP), Poder Judiciário (PJ) e as equipes multiprofissionais das políticas públicas de Assistência Social e de Saúde no estado do Ceará. A metodologia consistiu na análise quantitativa e qualitativa das demandas dos anos de 2013 e 2015 sobre as requisições do MP ou do PJ. Os resultados evidenciam que as requisições para as equipes de políticas públicas produzem sobrecarga de trabalho, conflitos éticos, bem como aumento das tensões entre equipes e população acompanhada. Concluiu-se que se faz necessário que o MP e o PJ formem suas equipes de apoio com profissionais peritos e assistentes técnicos, e que estes trabalhem em colaboração com os profissionais das políticas públicas, bem como se faz necessário o estabelecimento de diálogo interinstitucional para melhoria dos fluxos de colaboração.

1 Data de recebimento: 02/04/2018. Data de aceite: 18/06/2018.

2 Psicólogo (CRP 11/06632), Técnico de Referência e Servidor Público de Carreira do Centro de Referência de Assistência Social (CRAS) da Prefeitura Municipal de Umirim-CE. Conselheiro do Conselho Regional de Psicologia da $11^{\text {a }}$ Região (CRP 11), gestões 2013-2016 e 2016-2019. Mestre em Saúde da Família pela Universidade Federal do Ceará (UFC) / Fundação Oswaldo Cruz (FIOCRUZ). Docente e pesquisador dos seguintes temas: Psicologia, Saúde Coletiva, Saúde da Família, Saúde Mental, Assistência Social, Políticas Públicas e Participação Popular. E-mail: diegomendoncaviana@gmail.com 
Palavras-chave: Políticas Sociais. Ministério Público. Poder Judiciário. Colaboração. Políticas Públicas.

\section{INTRODUÇÃO}

A colaboração interinstitucional entre o Ministério Público (MP), o Poder Judiciário (PJ) e as Políticas Públicas de Assistência Social e Saúde tem sido uma das temáticas que geram muitos conflitos e controvérsias no Estado do Ceará. Esta afirmação inicial está embasada nos dados oriundos de um estudo realizado pelo Conselho Regional de Psicologia da $11^{a}$ Região - Ceará (CRP 11) em parceria com o grupo de trabalho "Demandas de Justiça aos Profissionais Psicólogos em Políticas Públicas" do Conselho Federal de Psicologia (CFP) entre os anos de 2013 e 2015.

O resultado do citado levantamento de informações estruturantes serviu de fundamento para a elaboração do parecer técnico intitulado "Parecer a Respeito de Pedido Oriundos do Ministério Público e do Poder Judiciário aos Profissionais das políticas públicas de Assistência Social e de Saúde - orientações e reflexões", de autoria do CRP 11 no ano de 2015, bem como a elaboração de uma normativa sobre o assunto chamada "Resolução CRP 11 nº2/2016, cuja ementa é a seguinte: disciplina e estabelece critérios e regras para notificação ao Conselho Regional de Psicologia da $11^{\text {a }}$ Região por parte dos psicólogos inscritos neste regional, quando receberem demandas do Sistema de Justiça, do Ministério Público e órgãos afins e dá outras providências.

Como desdobramento deste debate, o artigo pretende apresentar as problemáticas que dizem respeito, direta ou indiretamente, a todas as profissões que trabalham nas políticas públicas de Assistência Social e de Saúde, bem como trazer dados e elementos que possam contribuir para a melhoria dos processos de colaboração interinstitucional entre o Ministério Público, o Poder Judiciário e os profissionais das mencionadas políticas públicas de diversos mu- 
nicípios do Estado do Ceará. Serão abordados os aspectos legais, éticos, técnicos e políticos dessa conjuntura, a partir da Psicologia como ciência e profissão, contudo, o mérito pode ser ampliado para o debate das demais profissões que atuam em conjunto, em virtude de as questões serem semelhantes por analogia técnica.

\section{METODOLOGIA}

Este estudo se originou de uma proposta de pesquisa com dados qualitativos e quantitativos articulados em termos de abordagem, possuindo caráter aplicado quanto à sua natureza, exploratório quanto aos objetivos e de caráter longitudinal quanto ao tempo. Destaca-se, ainda, que o estudo possuiu características de pesquisa documental e pesquisa de levantamento quanto aos seus procedimentos de acordo com os entendimentos consolidados nos estudos metodológicos de Silveira e Córdova (2009). Os dados aqui citados foram obtidos por meio do levantamento sistemático das dúvidas enviadas por correio eletrônico pelos profissionais ao Conselho Regional de Psicologia da 11 a Região entre os anos de 2013 e 2015.

Os sujeitos do citado levantamento foram psicólogos (as) devidamente inscritos(as) no CRP 11, que possuíam atuação direta ou indireta nas políticas públicas de Assistência Social ou Saúde nos municípios do Estado do Ceará, e que tinham recebido requisições formais do Ministério Público, do Poder Judiciário, do Conselho Tutelar, dos órgãos de Polícia e serviços afins.

O envio de dados para o estudo ocorreu por convite público do CRP 11, de forma voluntária, respeitando os preceitos da confidencialidade e sigilo da legislação em vigência. Como se tratava de levantamento de interesse restrito para fins de conselho de profissão, os dados coletados serviram para orientação interna das políticas do CRP 11, e para orientação da categoria com a confecção de pareceres e documentos técnicos, com disposição apenas dos dados descritivos e 
suprimindo a fonte das informações para resguardar o sigilo.

Com a égide na nova regulamentação sobre pesquisa com seres humanos, mais especificamente, a RESOLUÇÃO No 510, DE 07 DE ABRIL DE 2016 do Conselho Nacional de Saúde, tornou-se possível disponibilizar estes dados por meio de publicações, resguardadas as garantias de sigilo.

Os dados coletados foram tabulados e categorizados de acordo com cada área temática segundo o tipo de requisição feita aos profissionais de Psicologia nas políticas públicas em questão. As informações quantitativas foram tratadas sob o prisma da estatística descritiva básica e os dados qualitativos foram analisados sob a compreensão da análise de conteúdo de Bardin (1977).

Após a organização das informações, foi realizado extenso levantamento de legislações e materiais técnicos pertinentes da profissão de Psicologia e das políticas públicas de Assistência Social e de Saúde. O confronto dos dados com os postulados da legislação e da literatura permitiram a elaboração deste artigo e dos documentos técnicos de orientação para os profissionais e para os gestores das políticas públicas.

\section{RESULTADOS E DISCUSSÃO}

\subsection{Aspectos Legais, Éticos e Técnicos da Colaboração Interinstitucional}

Um dos primeiros pressupostos que precisam ficar evidentes neste texto diz respeito ao fato de que os profissionais de Psicologia, e das demais profissões que integram equipes multiprofissionais das políticas públicas de Assistência Social, de Saúde e das demais políticas setoriais tem por dever colaborar com o Ministério Público, com o Poder Judiciário e com o Sistema de Garantia de Direitos em vigência no país. Esse pressuposto está amplamente sedimentado nas deter- 
minações legais contidas nos seguintes dispositivos: "arts. 28, §5; $46, \S 4^{\circ} ; 50, \S \S 3^{\circ}$ e $4^{\circ} ; 166, \S 7^{\circ}$ e $197-C$, $2^{\circ}$, todos da Lei no 8.069/90, assim como nos arts. $42, \S 1^{\circ} ; 53 ; 57$, caput; 58 e 64 , todos da Lei $n^{\circ}$ 12.594/2012" (DIGIÁCOMO, 2014. p 03). Além destes dispositivos citados, há ampla previsão de colaboração na Constituição Federal de 1988, bem como em um arcabouço amplo de legislações, que visam a proteger a sociedade da violação de direitos fundamentais. Ou seja, os profissionais das políticas públicas citadas são os responsáveis pelo acompanhamento cotidiano de indivíduos e famílias e, por tal razão, podem contribuir com importantes informações e opiniões técnicas em processos que visem à proteção coletiva e individual de direitos.

Nesse sentido, não se está a discutir se deve ou não haver a colaboração entre profissionais das políticas públicas, o Ministério Público e o Poder Judiciário. O que se discute, de forma muito contundente e séria, é como esta colaboração poderá ocorrer de forma a preservar as prerrogativas profissionais e os direitos civis que a Lei brasileira visa a proteger. No caso específico da Psicologia, há previsão deste tipo de colaboração no Código de Ética Profissional do Psicólogo (Resolução CFP n 10/2005).

Nota-se que a colaboração em juízo e em situações análogas está prevista em Código de Ética e em legislações correlatas. Em tais termos, a colaboração não pode induzir a quebra de preceitos éticos da profissão. O dever de colaborar é resguardado, contudo cabem alguns questionamentos sobre os procedimentos adotados nos pedidos e requisições aos profissionais. Os citados questionamentos versam a respeito dos prazos desarrazoados, impertinência dos recursos solicitados, bem como invasão de competência técnica por parte dos operadores do Direito na determinação de métodos e técnicas, que não lhes cabem para os profissionais das políticas públicas.

Apesar de haver previsão de colaboração dos profissionais das políticas públicas com o Ministério Público, com o Poder Judiciário e órgãos afins, as equipes multiprofissionais das políticas públicas 
possuem atribuições específicas tipificadas que, em muitos casos, entram em choque com as requisições do Sistema de Justiça.

Nesse sentido, na política de Assistência Social, de acordo com as recomendações contidas para a Proteção Social Básica (PSB), em especial para a tipificação e caracterização dos serviços ofertados pelas equipes profissionais dos Centros de Referência da Assistência Social (CRAS), elaboradas pelo Ministério do Desenvolvimento Social e Combate à Fome (MDS), tem-se os seguintes termos importantes:

\begin{abstract}
Qual(is) a(s) função(ões) do CRAS?
Representa a principal estrutura física local para a proteção social básica, desempenha papel central no território onde se localiza, possuindo a função exclusiva da oferta pública do trabalho social com famílias por meio do serviço de Proteção e Atendimento Integral a Famílias (PAIF) e gestão territorial da rede socioassistencial de proteção social básica. (grifos do autor).
\end{abstract}

A respeito das equipes que trabalham com a proteção social especial (PSE) na política de Assistência Social, em especial para a tipificação e caracterização dos serviços ofertados pelas equipes profissionais dos Centros de Referência Especializado de Assistência Social (CREAS), elaboradas pelo Ministério do Desenvolvimento Social e Combate à Fome (MDS), e contidas na publicação "Orientações Técnicas: Centro de Referência Especializado de Assistência Social CREAS (2011)", tem-se os seguintes termos importantes:

Frente ao exposto, e considerando o papel do CREAS e competências decorrentes, destaca-se que a este não cabe: - Ocupar lacunas provenientes da ausência de atendimentos que devem ser ofertados na rede pelas outras políticas públicas e/ou órgãos de defesa de direito;

- Ter seu papel institucional confundido com o de outras políticas ou órgãos, e, por conseguinte, as funções de sua equipe com as de equipes interprofissionais de outros atores da rede, como, por exemplo, da segurança pública (Delegacias Especializadas, unidades do sistema prisional, etc), órgãos de defesa e responsabilização (Poder Judiciário, Ministério Pú- 
blico, Defensoria Pública e Conselho Tutelar) ou de outras políticas (saúde mental, etc.) (grifos do autor).

Na política de Saúde, na atenção primária/básica, considerando o disposto na publicação CADERNOS DE ATENÇÃO BÁSICA. NÚCLEO DE APOIO À SAÚDE DA FAMÍLIA - VOLUME I. FERRAMENTAS PARA GESTÃO E PARA O TRABALHO COTIDIANO do Ministério da Saúde, ano 2014, existe o seguinte entendimento sobre o processo de trabalho das equipes do NASF na relação com a rede de atenção:

\begin{abstract}
O Nasf, portanto, faz parte da Atenção Básica, mas não se constitui como um serviço com espaço físico independente. Isso quer dizer que os profissionais do Núcleo utilizam-se do próprio espaço das Unidades Básicas de Saúde e do território adstrito para o desenvolvimento do seu trabalho. Eles atuam a partir das demandas identificadas no trabalho conjunto com as equipes vinculadas, de forma integrada à Rede de Atenção à Saúde e seus serviços, além de outras redes como o Sistema Único da Assistência Social (Suas), redes sociais e comunitárias.
\end{abstract}

Em 2017, a legislação dos NASFs foi atualizada por meio da PORTARIA No 2.436, DE 21 DE SETEMBRO DE 2017 do Ministério da Saúde. Neste documento, tanto a nomenclatura quanto as atribuições foram redimensionadas da seguinte forma:

Compete especificamente à Equipe do Núcleo Ampliado de Saúde da Família e Atenção Básica (Nasf- AB):

a) Participar do planejamento conjunto com as equipes que atuam na Atenção Básica à que estão vinculadas;

b) Contribuir para a integralidade do cuidado aos usuários do SUS principalmente por intermédio da ampliação da clínica, auxiliando no aumento da capacidade de análise e de intervenção sobre problemas e necessidades de saúde, tanto em termos clínicos quanto sanitários; e

c) Realizar discussão de casos, atendimento individual, compartilhado, interconsulta, construção conjunta de projetos terapêuticos, educação permanente, intervenções no território e na saúde de grupos populacionais de todos os ciclos de vida, e da coletividade, ações intersetoriais, ações de pre- 
venção e promoção da saúde, discussão do processo de trabalho das equipes dentre outros, no território. (grifos do autor).

No caso da política de Saúde Mental, considerando o disposto na PORTARIA No 3.088, DE 23 DE DEZEMBRO DE 2011, que institui a Rede de Atenção Psicossocial para pessoas com sofrimento ou transtorno mental e com necessidades decorrentes do uso de crack, álcool e outras drogas, no âmbito do Sistema Único de Saúde (SUS), em especial, nos termos dispostos no Art. $4^{\circ}$, Art. $7^{\circ}$ e seus dispositivos tem-se a seguinte determinação:

\begin{abstract}
Art. $4^{\circ}$ São objetivos específicos da Rede de Atenção Psicossocial:

I promover cuidados em saúde especialmente para grupos mais vulneráveis (criança, adolescente, jovens, pessoas em situação de rua e populações indígenas);

Art. $7^{\circ} \mathrm{O}$ ponto de atenção da Rede de Atenção Psicossocial na atenção psicossocial especializada é o Centro de Atenção Psicossocial.

$\S 2^{\circ}$ As atividades no Centro de Atenção Psicossocial são realizadas prioritariamente em espaços coletivos (grupos, assembleias de usuários, reunião diária de equipe), de forma articulada com os outros pontos de atenção da rede de saúde e das demais redes.

$\S 3^{\circ} \mathrm{O}$ cuidado, no âmbito do Centro de Atenção Psicossocial é desenvolvido por intermédio de Projeto Terapêutico Individual, envolvendo em sua construção a equipe, o usuário e sua família, e a ordenação do cuidado estará sob a responsabilidade do Centro de Atenção Psicossocial ou da Atenção Básica, garantindo permanente processo de cogestão e acompanhamento longitudinal do caso. (grifos do autor).
\end{abstract}

A simples análise dos subsídios acima mencionados permitem concluir, de forma cristalina, que as equipes dos CRAS e dos CREAS na Política de Assistência Social e as Equipes de NASFs-AB e CAPS na Política de Saúde possuem as prerrogativas de atuação com indivíduos e famílias, em uma perspectiva comunitária, longitudinal, integral e articulada com os demais serviços. Um dos pressupostos do 
trabalho desenvolvido é o estabelecimento de vínculos e de relações de confiança entre equipes e comunidade. Este tipo de trabalho é majoritariamente incompatível técnica e eticamente ${ }^{3}$ com o trabalho pericial ou de emissão de opinião técnica em situações de litígio requeridas pelo Ministério Público e pelo Poder Judiciário. Importante esclarecer que as citadas equipes podem e devem colaborar, fornecendo as informações gerais e complementares do acompanhamento realizado para contextualizar os órgãos do sistema de garantia de direitos. Contudo, este tipo de colaboração não pode ser confundido com o papel de assistente técnico ou perito costumeiramente requerido pelo Ministério Público e pelo Poder Judiciário.

Um dos argumentos parcialmente contrários existentes a respeito desta problemática consiste na linha de questionamento proferida pelo documento Nota Técnica $N^{\circ}$ 03/2018, de autoria do Centro de Apoio Operacional às Promotorias de Justiça de Defesa da Infância e Juventude (Caop Infância e Juventude) do Ministério Público Estadual de Pernambuco (MPPE) em 16/05/2018. Nessa nota, o MPPE argumenta, em síntese, que os profissionais de Psicologia devem quebrar o sigilo e situações em que haja indícios ou provas de fato de violação de direitos, e que as profissões não possuem autonomia de preservação na confidencialidade, quando isto for de encontro ao interesse público previsto em Lei.

Importante salientar que este artigo argumenta que o sigilo profissional não é absoluto, bem como que pode e deve haver colaboração entre os profissionais e o sistema de justiça como um todo para a preservação dos direitos fundamentais e da cidadania. Contudo, a referida Nota Técnica, que traz uma importante elucidação das legislações sobre a matéria e traz boas sugestões sobre o estabelecimento de fluxos da colaboração interinstitucional, não discutiu em detalhes quanto aos possíveis conflitos éticos que podem ocorrer neste processo. Nesse sentido, não caberia ao MP apenas afirmar

3 Vedação prevista no Art. 20, alíneas "J" e "K" do Código de Ética Profissional do Psicólogo. 
que os profissionais devem romper administrativamente com a confidencialidade sem que haja uma proposta de formação de equipes do próprio sistema de justiça para avaliar a condução posterior do processo diante do iminente conflito ético ao qual os profissionais ficarão expostos após rompimento de sigilo. Cabe considerar, em nível de sugestão, que a construção conjunta de documentos conjuntos entre o Sistema de Justiça e os Conselhos Regionais e Federal de Psicologia seja um caminho mais produtivo para sanar algumas controvérsias nesta temática. Em desdobramento a esta discussão, torna-se interessante considerar os elementos trazidos nas próximas seções deste artigo.

\subsection{Panorama das Requisições Feitas pelo Ministério Público e pelo Poder Judiciário aos Profissionais de Psicologia das Políticas Públicas no Ceará (2013-2015).}

De acordo com os dados do levantamento feito pelo CRP 11, a maior concentração de pedidos/demandas oriundas do Poder Judiciário, Ministério Público, Delegacias e órgãos dessa natureza encontram-se direcionadas para a Política de Assistência Social. Mais especificamente, 55, 17\% das demandas são direcionadas às equipes do Centro de Referência Especializado de Assistência Social (CREAS), enquanto 20,68\% das referidas demandas são dirigidas aos Centros de Referência da Assistência Social (CRAS). As demais porcentagens encontram-se distribuídas, espaçadamente, nas demais políticas, sendo os profissionais vinculados à política de saúde ocupantes do segundo lugar na quantidade de solicitações concentradas. Na política de saúde, predominam as solicitações ao Centro de Atenção Psicossocial (CAPS), com 3,44\% do total de solicitações das quais o CRP 11 tomou conhecimento. Abaixo segue a apresentação gráfica sobre os tipos de requisição mais frequentes aos psicólogos por parte do MP e PJ. 
Gráfico da Tipificação e Quantificação de Demandas Judiciais aos Psicólogos no Estado do Ceará (2013-2015)

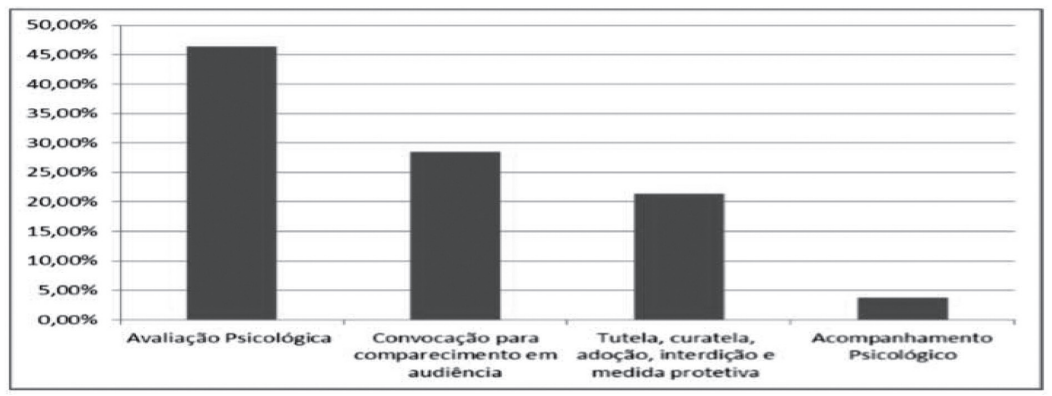

Fonte: Elaboração própria. Dados do Levantamento "Demandas de Justiça aos Profissionais Psicólogos em Políticas Públicas". CRP 11 (2015).

Diante do exposto, pelos dados do levantamento elaborado pelo CRP 11, existem evidências concretas de que os órgãos do Poder Judiciário, do Ministério Público e, em alguns casos, do Conselho Tutelar tem contribuído significativamente para mudança de orientação, do ponto de vista negativo4, de processo de trabalho das políticas públicas no Estado do Ceará. Estes fatos têm favorecido a sobrecarga das equipes profissionais das políticas públicas, pois as equipes possuem condições precárias de trabalho, e de exercício profissional para dar conta das demandas já pertencentes à sua rotina de trabalho regular e, ainda, recebem demandas extras do Ministério Público e do Poder Judiciário.

O contexto acima torna-se agravado pelo fato de que as requisições estipulam prazos exíguos para o cumprimento do solicitado, sendo os referidos prazos acompanhamentos de expressões admo-

4 Aumento de demandas de avaliação psicológica de caráter clinico, pericial e opiniões técnicas cujo objeto foge das atribuições profissionais para os quais os profissionais foram contratados nas políticas públicas de Assistência Social e de Saúde.,contribuindo, assim, para um desvio de finalidade das funções profissionais. 
estadoras tais como "sob pena de crime de desobediência", "improbidade administrativa", "prisão" e outros. Este tipo de requisição desarrazoada, além de sobrecarregarem o cotidiano das políticas públicas, contribuem para a instauração de ambiente de tensão entre os profissionais, as famílias acompanhadas e os órgãos requisitantes. Vale destacar que o próprio Conselho Nacional de Justiça (CNJ), no Provimento $N^{\circ} 36$ é preciso quanto à vedação de uso de expressões admoestadoras, quando se requisita apoio das políticas públicas do poder executivo.

Nota-se que promotores (as) e magistrados (as), por vezes, solicitam avaliações técnicas inadequadas, inclusive indicando os métodos supostamente mais adequados para que os profissionais realizem as atividades. Operadores do Direito não possuem competência para indicar métodos técnicos por duas razões latentes: a) esta prática dos operadores do Direito constitui invasão de competência técnica das demais profissões regulamentadas; b) os profissionais que acompanham as demandas dos indivíduos e famílias, no cotidiano, possuem autoridade e competência técnica para estipular o método e os procedimentos necessários e mais adequados com vistas ao benefício do acompanhamento.

As requisições podem conduzir para situações de flagrante conflito ético dos profissionais. Esses conflitos são percebidos quando os profissionais são requeridos para exercerem tarefas típicas de peritos, assistentes técnicos, ou funções semelhantes nas quais precisam emitir avaliações para situações litigiosas das famílias das quais há vínculo de acompanhamento formado. Esse vínculo pode ser rompido com as famílias e de forma irreversível, após a realização de algumas requisições do Ministério Público e do Poder Judiciário. Por esse motivo, o CRP 11, fundamentado nos termos da Resolução CRP $11 n^{\circ}$ 02/2016, tem orientado os profissionais que, ao avaliarem risco de conflito ético, respondam ao MP e aos Magistrados as razões de impossibilidade, sempre deixando a autarquia de classe 
informada dos fatos. Orientação semelhante é válida sobre as questões de quebra de sigilo nos termos do Código de Ética Profissional do Psicólogo, quando forem requeridas pelo Ministério Público e pelo Poder Judiciário.

\section{CONCLUSÃO}

Diante dos elementos apresentados neste artigo, evidenciou-se que a colaboração interinstitucional entre Ministério Público, Poder Judiciário e os profissionais das políticas públicas de Assistência Social e Saúde pode ocorrer de forma coerente e dialogada. A colaboração com o sistema de garantia de direitos por parte das equipes multiprofissionais das políticas públicas possui vasta previsão legal, bem como atende aos preceitos da proteção integral de indivíduos e famílias que se encontrem em situação de violações de direitos ou de risco à dignidade e à vida.

Conclui-se, também, pelos dados elencados que as requisições do Ministério Público e do Poder Judiciário produzem sobrecarga de trabalho para os trabalhadores das políticas públicas, pois estes trabalhadores, além de realizarem suas funções primordiais do cotidiano dos serviços, recebem muitas demandas extras do MP e PJ, com prazos exíguos e exigências questionáveis nos despachos. Defende-se neste artigo, e em todos os materiais técnicos a respeito dessa temática, que o Ministério Público e o Poder Judiciário abram concurso público para formação de seus quadros de assistentes técnicos e peritos. Na impossibilidade de abertura de concurso, que se realizem seleções públicas específicas para credenciamento de profissionais com as citadas funções. Desta forma, os profissionais das políticas públicas contribuiriam para qualificar, em caráter complementar e contextual, o trabalho dos peritos e assistentes técnicos do quadro de servidores do MP e do PJ.

Por fim, urge que seja estabelecido um diálogo construtivo entre 
o Poder Executivo, o Poder Judiciário e o Ministério Público, para que a colaboração interinstitucional ocorra de forma coerente, tecnicamente adequada e eticamente respaldada na garantia dos direitos de indivíduos e famílias nos diversos municípios do Estado do Ceará.

\section{INSTITUTIONAL COLLABORATION BETWEEN PUBLIC PRO- SECUTORS, JUDGES AND PSYCHOLOGY PROFESSIONALS OF PUBLIC POLICIES FOR SOCIAL AND HEALTH ASSISTANCE - ETHICAL LIMITS AND POSSIBILITIES}

\section{ABSTRACT}

The objective of this study is to discuss the various complex factors involved in the interinstitutional collaboration between the Public Prosecutor's Office (MP), the Judiciary Branch (PJ) and the multi-professional teams of the Public Social Assistance and Health policies in the state of Ceará. The methodology consisted in the quantitative and qualitative analysis of the demands of the years of 2013 and 2015 on the requests of the MP or the PJ. The results show that the requisitions for the public policy teams produce work overload, ethical conflicts, as well as increased tensions between teams and the population monitored. It was concluded that it is necessary that the MP and the PJ form their support teams with professional experts and technical assistants and that they work in collaboration with public policy professionals, as well as the establishment of interinstitutional dialogue to improve flows of collaboration.

Keywords: Social Policies. Public ministry. Judicial power. Collaboration. Public policy. 


\section{REFERÊNCIAS}

BARDIN, L. Análise de conteúdo. Lisboa: Edições 70; 1977.

BRASIL. LEI No 12.594, DE 18 DE JANEIRO DE 2012 que institui o Sistema Nacional de Atendimento Socioeducativo (Sinase), regulamenta a execução das medidas socioeducativas destinadas a adolescente que pratique ato infracional. Brasília, 2012.

. LEI No 8.069, DE 13 DE JULHO DE 1990 que dispõe sobre o Estatuto da Criança e do Adolescente e dá outras providências. Brasília, 1990. MINISTÉRIO DA SAÚDE. PORTARIA No 2.436, DE 21 DE SETEMBRO DE 2017 que aprova a Política Nacional de Atenção Básica, estabelecendo a revisão de diretrizes para a organização da Atenção Básica, no âmbito do Sistema Único de Saúde (SUS). Brasília: MS, 2017.

MINISTÉRIO DA SAÚDE. PORTARIA No 3.088, DE 23 DE DEZEMBRO

DE 2011 que institui a Rede de Atenção Psicossocial para pessoas com sofrimento ou transtorno mental e com necessidades decorrentes do uso de crack, álcool e outras drogas, no âmbito do Sistema Único de Saúde (SUS). Brasília: MS, 2011.

. MINISTÉRIO DA SAÚDE. Secretaria de Atenção à Saúde. Departamento de Atenção Básica. Núcleo de Apoio à Saúde da Família / Ministério da Saúde, Secretaria de Atenção à Saúde, Departamento de Atenção Básica. - Brasília: Ministério da Saúde, 2014. 116 p.: il. - (Cadernos de Atenção Básica, n. 39).

MINISTÉRIO DO DESENVOLVIMENTO SOCIAL E COMBATE À FOME.

Orientações Técnicas: Centro de Referência de Assistência Social CRAS/ Ministério do Desenvolvimento Social e Combate à Fome. - 1. ed. Brasília: Ministério do Desenvolvimento Social e Combate à Fome, 2009. 72 p. MINISTÉRIO DO DESENVOLVIMENTO SOCIAL E COMBATE À FOME.

Orientações Técnicas: Centro de Referência Especializado de Assistência Social - CREAS/Ministério do Desenvolvimento Social e Combate à Fome. - 1. ed. - Brasília: Ministério do Desenvolvimento Social e Combate à Fome/ Gráfica e Editora Brasil LTDA, 2011. 120 p.

CONSELHO FEDERAL DE PSICOLOGIA. RESOLUÇÃO CFP n 10/2005 que Aprova o Código de Ética Profissional do Psicólogo. Brasília: CFP, 2005. 
CONSELHO NACIONAL DE JUSTIÇA. Provimento $\mathrm{N}^{\circ} 36$ que dispõe sobre a estrutura e procedimentos das varas da infância e juventude. Brasília: CNJ, 2014.

CONSELHO NACIONAL DE SAÚDE. RESOLUÇÃO No 510, DE 07 DE ABRIL DE 2016 que dispõe sobre as normas aplicáveis a pesquisas em Ciências Humanas e Sociais cujos procedimentos metodológicos envolvam a utilização de dados diretamente obtidos com os participantes ou de informações identificáveis ou que possam acarretar riscos maiores do que os existentes na vida cotidiana, na forma definida nesta Resolução. Brasília, 2016.

CONSELHO REGIONAL DE PSICOLOGIA DA $11^{\text {a }}$ REGIÃO (CRP 11). Parecer a Respeito de Pedido Oriundos do Ministério Público e do Poder Judiciário aos Profissionais das políticas públicas de Assistência Social e de Saúde - orientações e reflexões. Fortaleza: CRP 11, 2015.

DIGIÁCOMO, M. J. MINISTÉRIO PÚBLICO DO PARANÁ. Ofício n 307/2014SEC. Parecer do Fórum Regional de Assistência Social contra as requisições de atendimento e envios de relatórios efetuadas pelo Ministério Público e Poder Judiciário. Curitiba: MPPR, 2014. Disponível em: < http://www.crianca.mppr.mp.br/arquivos/File/parecer/creas_recusa_no_envio_de_relatorios_parecer.doc > Acesso em 23 de março de 2018. SILVEIRA, D.T; CÓRDOVA, F.P. Unidade 2 - A pesquisa científica. IN: GERHARDT, E;

SILVEIRA, D.T (Org.). Métodos de pesquisa. Porto Alegre: Editora da UFRGS, 2009. 